\title{
Surface-Associated Heat Shock Proteins of Legionella pneumophila and Helicobacter pylori: Roles in Pathogenesis and Immunity
}

\author{
P.S. Hoffman ${ }^{1 *}$ and R.A. Garduno ${ }^{2}$ \\ ${ }^{1}$ Department of Microbiology and Immunology, Dalhousie University, Halifax, Nova Scotia, Canada \\ ${ }^{2}$ Division of Infectious Diseases, Department of Medicine, Dalhousie University, \\ Halifax, Nova Scotia, Canada
}

\begin{abstract}
Bacterial heat shock proteins (Hsps) are abundantly produced during the course of most microbial infections and are often targeted by the mammalian immune response. While Hsps have been well characterized for their roles in protein folding and secretion activities, little attention has been given to their participation in pathogenesis. In the case of Legionella pneumophila, an aquatic intracellular parasite of protozoa and cause of Legionnaires' disease, Hsp60 is uniquely located in the periplasm and on the bacterial surface. Surface-associated Hsp60 promotes attachment and invasion in a HeLa cell model and may alter an early step associated with the fusion of phagosomes with lysosomes. Avirulent strains of $L$. pneumophila containing defined mutations in several dot/icm genes are defective in localizing $\mathrm{Hsp} 60$ onto their surface and are reduced approximately 1000 -fold in their invasiveness towards HeLa cells. For the ulcer-causing bacterium Helicobacter pylori, surfaceassociated Hsp60 and Hsp70 mediate attachment to gastric epithelial cells. The increased expression of these Hsps following acid shock correlates with both increased association with and inflammation of the gastric mucosa. A role for Hsps in colonization, mucosal infection and in promoting inflammation is discussed. Infect. Dis. Obstet. Gynecol. 7:58-63, 1999. @1999 Wiley-Liss, Inc.
\end{abstract}

KEY WORDS

Legionella pneumophila; Helicobacter pylor; heat shock proteins; cellular immunity; adhesion

$\mathrm{H}_{\mathrm{os}}^{\mathrm{e}}$ eat shock proteins (Hsps, also known as stress or chaperone proteins), particularly the members of the Hsp60 and Hsp70 families, have been recognized for years as immunodominant antigens of many microbial pathogens. ${ }^{1-3}$ Moreover, immune responses against bacterial Hsps have often been implicated in certain autoimmune diseases ${ }^{2,3}$ and chronic infections. ${ }^{4,5}$ Yet, autoimmune disease can be prevented in several animal model systems by vaccination with $\mathrm{Hsps},{ }^{6,7}$ indicating that the immune response to Hsps is both complex and can be modulated by extrinsic factors. Generally, immu- nodominant proteins are thought of as those that are either secreted or expressed on the surface of microbes, so studies indicating that the Hsp60 of Escherichia coli (GroEL) is exclusively located in the cytoplasm seemed contradictory. In addition, none of the heat shock or stress regulated genes studied to date encode proteins with signal sequences or any other known secretion-specific motif to suggest these proteins are secreted. Therefore, early reports implicating Hsps in pathogenesis were not taken too seriously or were thought to be too preliminary to support a strong case. To begin

Grant sponsor: Medical Research Council of Canada; Astra Pharma, Canada; Natural Sciences Engineering Council of Canada.

*Correspondence to: Dr. Paul S. Hoffman, Department of Microbiology \& Immunology, Sir Charles Tupper Building, Rm 7P, Dalhousie University, Halifax, Nova Scotia, Canada B3H 4H7. E-mail: phoffman@tupdean2.med.dal.ca 

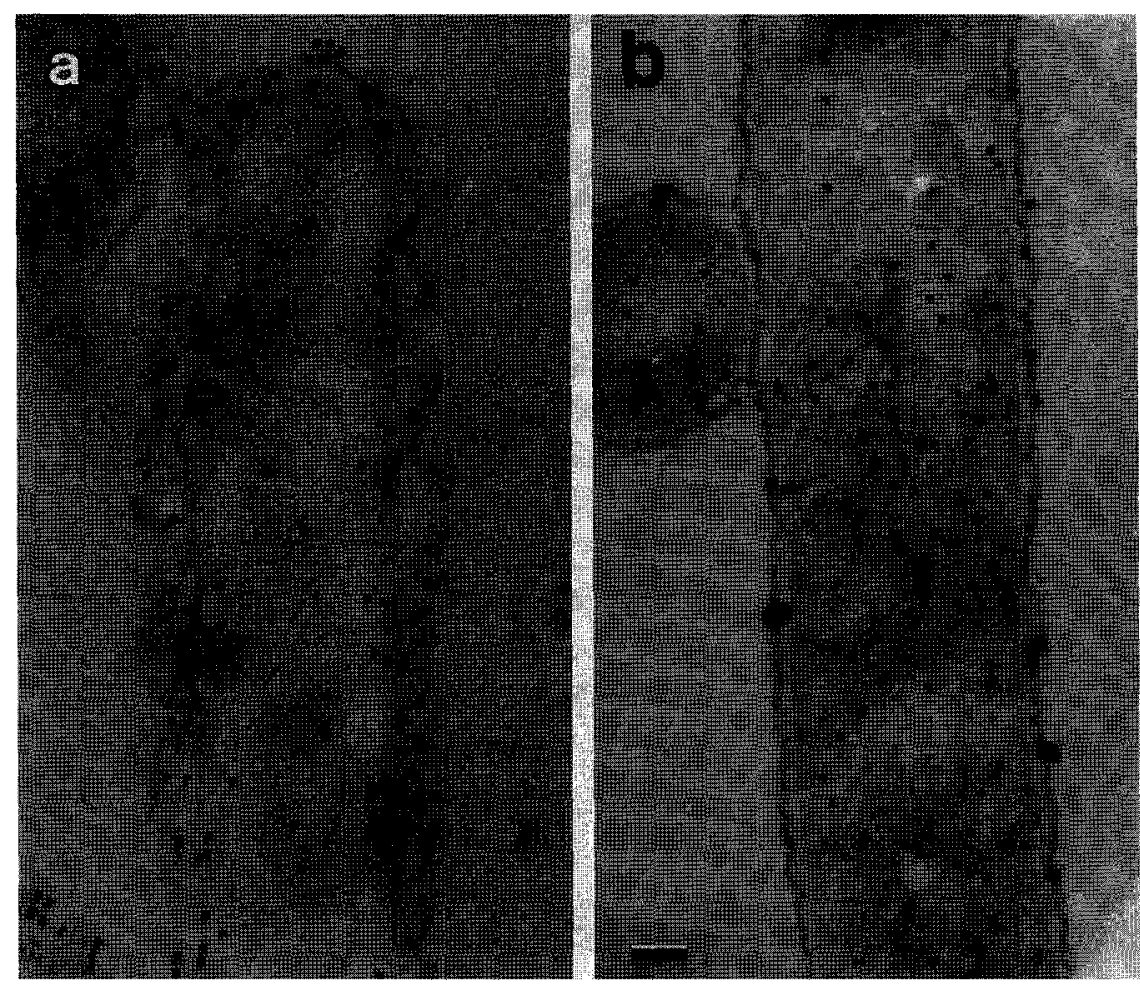

Fig. I. Immunolocalization of $\mathrm{Hsp} 60$ in L. pneumophila (a) and E. coli expressing recombinant L. pneumophila Hsp60 from pSHI 6 (b). Bar indicates $0.1 \mathrm{~mm}$ for both panels.

to understand what roles Hsps might play in pathogenesis and host immune response, we have been investigating Legionella pneumophila, an aquatic microbe that has not evolved with humans, and Helicobacter pylori, an organism highly evolved and specialized to the human gastric mucosa. While both pathogens express Hsps on their surface, the pathogenesis role for these Hsps differs. Our studies suggest that the diversity of function attributed to Hsps, and in particular to pathogenesis mechanisms, is largely determined by cellular location.

\section{LOCALIZATION OF HSPS}

Immunolocalization techniques have been successfully used to determine the cellular location of Hsps. Surface localization can be determined with whole bacterial cells by either immunofluorescence (light microscopy) or by immunogold electron microscopy using monoclonal (MAb) or polyclonal (PAb) antibodies raised against the particular Hsp. Performing immunolocalization with sectioned bacteria permits further localization of these proteins among internal compartments. When immunogold electron microscopy was performed on thin sections of $L$. pneumophila, using antibodies specific for Hsp60 and gold-conjugated anti-immunoglobulin (MAb or PAb), the gold particles were found to surround the periphery of the bacteria (see Fig. 1a), indicating both a periplasmic and a surface location. 8 ,9 As a control for these experiments, recombinant L. pneumophila $\mathrm{Hsp60}$ was produced from a clone in $E$. coli. As can be seen in Figure $1 \mathrm{~b}$, the E. coli bacteria accumulated Hsp60 in the cytoplasm, with little or no gold spheres located in other compartments or on the surface. Comparative immunogold studies with antibodies raised to well-known surface associated antigens (LPS, OmpS) gave results similar to those observed with $\mathrm{Hsp} 60$ antiserum, ${ }^{8}$ supporting the case that Hsp60 is a legitimate surface antigen in L. pneumophila. Several studies employing immunogold labeling of whole $H$. pylori cells showed that Hsp60 was located on the bacterial surface. ${ }^{10-12}$ Further, immunogold labeling of biopsy samples from patients infected with $H$. pylori also revealed Hsp60 associated with inflamed tissue. ${ }^{13}$ These studies indicate that Hsp60 is one of the dominant proteins expressed in situ by $H$. pylori.

An examination of Hsp60 sequences from many 


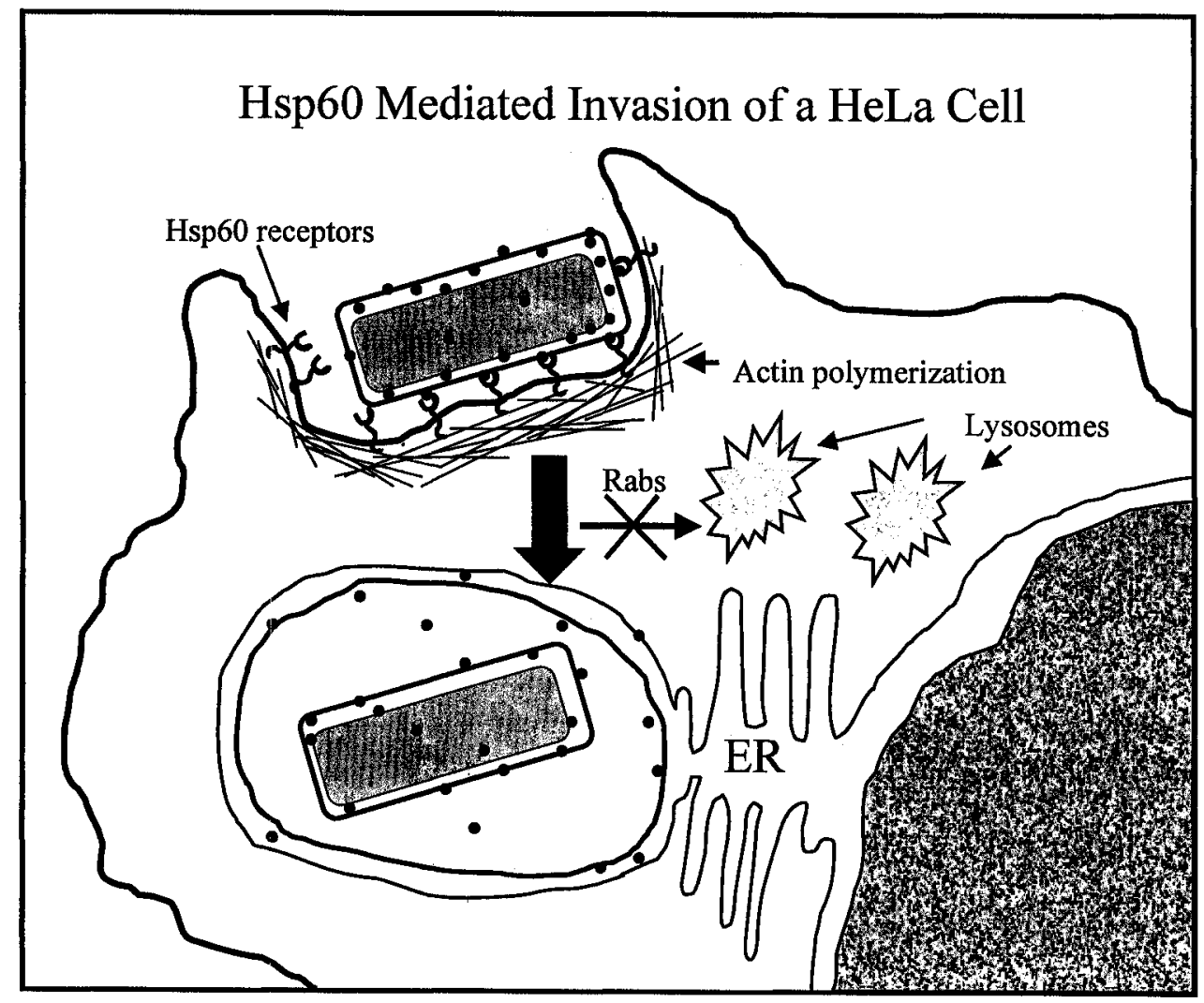

Fig. 2. Parasite-directed receptor-mediated endocytosis of $L$. pneumophila by HeLa cells. The illustration indicates that Hsp60 binds to specific receptors on the HeLa cells, initiating actin polymerization and endocytosis of the bacterium into an early endosome. We propose that Hsp60, interacting with the phagosome membrane, alters signal transduction networks that would ordinarily traffic the bac-

microbial pathogens deposited in Genbank reveals no obvious secretion motifs in this family of highly conserved proteins. Therefore, it has been proposed that the observed surface location of Hsps is achieved by mechanisms that do not involve secretion. For instance, it has been proposed that in $H$. pylori, Hsp60 released from naturally lysed bacteria binds to the surface of neighboring bacteria, ${ }^{11}$ but this is not the mechanism by which Hsp60 reaches the surface of $L$. pneumophila. ${ }^{8}$ Specific studies addressing the secretion of Hsp60 by L. pneumophila have not been conducted. However, the observation that $E$. coli expressing the L. pneumophila gene for Hsp60 does not secrete the protein would imply that $E$. coli lacks the machinery used by L. pneumophila and perhaps other bacteria to mobilize Hsp60 to the periplasm or cell surface. ${ }^{8}$

Avirulent mutants of L. pneumophila can be iso- teria-laden endosome to secondary lysosomes. A feature of the specialized endosome in which $L$. pneumophila replicates is its envelopment by the endoplasmic reticulum. "Rabs" indicates several families of GTP-proteins associated with trafficking activities. GTP = guanosine triphosphate; $\mathrm{ER}=$ endoplasmic reticulum.

lated in the laboratory by selection for tolerance to $0.65 \%$ sodium chloride. Mutations conferring sodium tolerance map to several regions of the chromosome containing the dot/icm genes involved in facilitating DNA or protein transport. ${ }^{14,15}$ These genes seem to be important in facilitating the surface localization of Hsp60 since avirulent mutants appear unable to efficiently localize Hsp60 to the surface. ${ }^{9}$ Several lines of evidence outlined in the following sections support the hypothesis that surface-exposed Hsp60 is utilized by L. pneumophila as an invasin that additionally may alter early events associated with organelle trafficking and phagolysosomal fusion.

\section{ADHERENCE AND INVASION}

Virulent as well as avirulent strains of $L$. pneumophila are phagocytized by professional phagocytes 


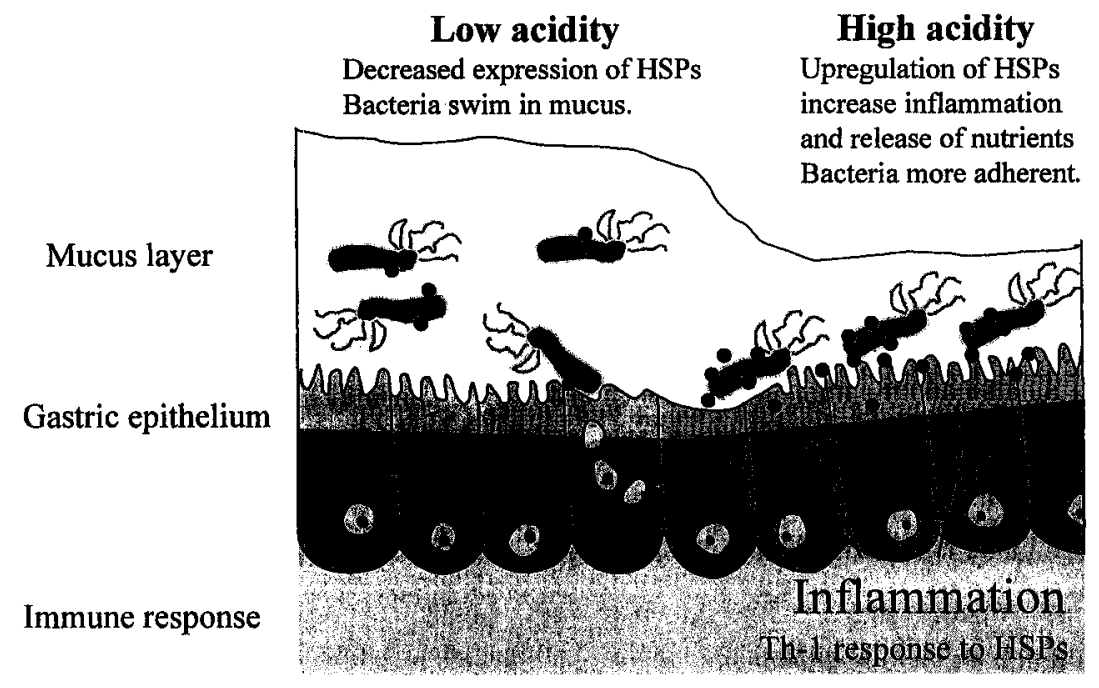

Fig. 3. Illustration of the interactions of $H$. pylori in the gastric mucosa. Under conditions of high acidity, such as might occur in a gastric or duodenal ulcer, $H$. pylori $H$ sps are upregulated and both localized on the surface and released. The increased surface-expressed Hsp60 and Hsp70 promote attachment to the epithelial cells, which might afford protection from the effects of high acid. Released Hsp 60 and $\mathrm{Hsp} 70$ promote inflammation through the stimulation

like macrophages, monocytes, neutrophils, and natural protozoan hosts. In all of these hosts, invasion cannot be easily dissected from abrogation of phagosome-lysosome fusion, since phagocytosis is host driven and the earliest distinguishable event in these cells occurs after internalization. In nonprofessional phagocytes, like epithelial cells (HeLa), invasion is generally parasite driven. Therefore, while virulent and avirulent strains of $L$. pneumophila are phagocytosed by macrophages with similar efficiencies, in HeLa, cells virulent strains of $L$. pneumophila are -1000 -fold more invasive than avirulent strains. Interestingly, avirulent strains of L. pneumophila were not impaired in their ability to adhere to HeLa cells. ${ }^{16}$ Using specific antibodies raised to $\mathrm{Hsp} 60$ or to the major outer membrane protein OmpS, we could demonstrate that while both anti-Hsp60 and anti-OmpS could block the association of virulent strains to HeLa cells, only the anti-OmpS serum blocked the association of avirulent L. pneumophila with HeLa cells. ${ }^{9}$ Collectively, these findings suggested that Hsp60 played a significant role in mediating invasion.

To further delineate the role of surfaceassociated $\mathrm{Hsp} 60$ in pathogenesis, competition of IL-Ib and IL- 12 production by recruited macrophages and interferon- $\gamma$ by activated Th-I T-cells. Under conditions of lower acid (more alkaline $\mathrm{pH}$ ), repression of Hsp production leads to less adherent bacteria that can be found throughout the mucus lining of the stomach. From this remote location, released Hsps can still promote inflammation, while the bacteria remain elusive to the mounting host immune response.

with purified Hsp60 or Hsp60 bound to latex beads was used to block attachment and invasion in the HeLa cell model..$^{9}$ Additionally, treatment of virulent, but not avirulent, L. pneumophila strains with trypsin was shown to degrade surface-associated Hsp60 and diminish invasiveness for HeLa cells. Finally, Hsp60 bound to latex beads, but not bovine serum albumin- (BSA) beads, were efficiently taken up by HeLa cells, and the phagosomes containing Hsp60-beads did not traffic to or fuse with secondary lysosomes as was observed for BSAbeads, suggesting that Hsp60 may interfere with early signals required for fusion with lysosomes. ${ }^{9}$ The notion that a single protein (Hsp60) mediates both invasion and abrogation of phagosomelysosome fusion is satisfying for three main reasons. First, previous studies had predicted that the invasion factor of $L$. pneumophila must be preexisting and not synthesized de novo, as invasion occurs in the presence of protein synthesis inhibitors. ${ }^{16} \mathrm{Sec}-$ ond, phagosome-lysosome fusion is an early event (virtually coupled with invasion) inhibited by virulent $L$. pneumophila even in the presence of protein synthesis inhibitors. Third, avirulent $L$. pneumophila strains that are unable to abrogate phago- 
some-lysosome fusion do not efficiently express Hsp60 on the cell surface. ${ }^{17}$

Our studies suggest that HeLa cells must express surface receptors specific for Hsps. The nature of surface receptors has not been fully explored but may be generic to the family of Hsps, since we showed that Hsp60-beads, but not BSAbeads, when bound to the surface of cytochalasin D-treated macrophages (frozen for phagocytosis) initiated the production of interleukin (IL)-1b via a protein kinase $\mathrm{C}$ signal transduction pathway. ${ }^{18}$ Since both Hsp60 and Hsp70 protein families can induce IL-1b and IL-12 production by macrophages, ${ }^{18,19}$ from the host defense perspective, these proteins most likely play a key role in immune recognition of invading microbes via a Th-1 mediated inflammatory response. Since Legionnaires' disease is a disease of the elderly and immunocompromised, one can appreciate the key role of the Th-1 immune response in immunity against intracellular pathogens like L. pneumophila.

Several groups have extensively explored the role of surface-located Hsps in H. pylori pathogenesis. Yamaguchi et al. have shown that Hsp60 mediates adhesion of $H$. pylori to human gastric epithelial cells. ${ }^{12}$ Treatment of $H$. pylori with an anti-Hsp60 monoclonal antibody dramatically decreased attachment of the bacteria to gastric epithelial cells. It should be noted that a number of adhesins have been described for $H$. pylori, and much more study is needed to delineate roles for these adhesins in $H$. pylori colonization and pathogenesis. Several groups have used immunogold electron microscopy to document the association of secreted $H$. pylori Hsp60 with inflamed gastric tissue. ${ }^{12,13}$ Studies in our group have shown that Hsp60 and Hsp70 can bind to glycosulfolipids. ${ }^{10,20}$ Recently, Hsp70 has been implicated in adhesion, and we suggested that Hsp70 might play a more important role in adhesion following acid stress. ${ }^{20}$ Our studies suggest that under acid stress, $H$. pylori bacteria exhibit elevated synthesis of Hsps and may be more adherent to mucosal epithelial cells. Under more alkaline conditions, such as might be generated by the action of the constitutively expressed urease, the bacteria may lower expression of Hsps, thus becoming less adherent and permitting the bacteria to migrate in the mucus to areas of higher acidity.

\section{CONCLUSIONS}

Our studies of two very different bacterial pathogens have revealed a range of activities for Hsps in pathogenesis. In the case of $L$. pneumophila, a pathogen whose pathogenesis strategy involves invading and growing in freshwater protozoa, Hsp60 appears to promote infection, as well as to participate in mediating an endosymbiotic relationship with its host. Surface-associated Hsp60 promotes attachment to macrophages and HeLa cells (see Fig. 2) through Hsp60 specific receptors that in the former signal production of IL-1b and IL-12, two important initiators of the cell mediated Th- 1 immune response. The production of interferon-g by the Th-1 immune response is critical to the resolution of the infection. ${ }^{18}$

H. pylori, a mucosal pathogen, has evolved a strategy of using Hsp60 and Hsp70 to promote attachment to gastric epithelial cells, particularly under conditions of high acidity (see Fig. 3). Liberated Hsps may also play a prominent role in promoting inflammation, since in a mouse model it has been shown that Hsps induce the production of interferon-g and tumor necrosis factor- $\alpha$ in gastric tissue infected with $H$. pylori (unpublished). We propose that the secretion of Hsps by the pathogen may be part of a colonization strategy and, by promoting inflammation, may provide a means for acquiring nutrients via the leakage of serum from inflamed tissue. Perhaps this strategy is plied by other mucosal pathogens, including Neisseria gonorrhea, ${ }^{21}$ Bordetella pertussis, ${ }^{8}$ Haemophilus ducreyi, ${ }^{22}$ and Coxiella burnetii, ${ }^{23}$ all of which seem to display Hsps on their cell surface. If indeed Hsps are "key signatures" for immune recognition of mucosal or intracellular pathogens, then it could be argued that normal flora like $E$. coli have learned to hide their Hsps (cytoplasmic location) from immune surveillance, and hence are tolerated ("no harm, no foul"). This knowledge may be helpful in the design of new vaccines or strategies to control autoimmune diseases.

\section{ACKNOWLEDGMENTS}

Portions of this work have been supported by grants to PSH from the Medical Research Council of Canada, Astra Pharma, Canada, and to RAG from the Natural Sciences and Engineering Council of Canada. 


\section{REFERENCES}

1. Young D, Lathigra R, Hendrix R, Sweetser D, Young RA. Stress proteins are immune targets in leprosy and tuberculosis. Proc Natl Acad Sci USA 1988;85:42674270.

2. Young RA, Elliott TJ. Stress proteins, infection, and immune surveillance. Cell 1989;59:5-8.

3. Kaufmann SHE. Heat shock proteins and the immune response. Immunol Today 1990;11:129-136.

4. Kligman I, Jeremias J, Rosenwaks Z, Witkin SS. Cellmediated immunity to human and Escherichia coli 60$\mathrm{kDa}$ heat shock protein in women: association with a history of spontaneous abortion and endometriosis. Am J Reprod Immunol 1998;40:32-36.

5. Domeika M, Domeika K, Paavonen J, Mardh PA, Witkin SS. Humoral immune response to conserved epitopes of Chlamydia trachomatis and human $60-\mathrm{kDa}$ heatshock protein in women with pelvic inflammatory disease. J Infect Dis 1998;177:714-719.

6. Van der Zee RS, Anderton M, Prakken AB, Paul AG, van $E$ den $W$. $T$ cell responses to conserved bacterial heat-shock-protein epitopes induce resistance in experimental autoimmunity. Semin Immunol 1998;10:35-41.

7. Bockova J, Elias D, Cohen IR. Treatment of NOD diabetes with a novel peptide of the hsp60 molecule induces Th2-type antibodies. J Autoimmun 1997;10:323329.

8. Garduno RA, Faulkner G, Trevors MA, Vats N, Hoffman PS. Immunolocalization of $\mathrm{Hsp} 60$ in Legionella pneumophila. J Bacteriol 1998;180:505-513.

9. Garduno RA, Garduno E, Hoffman PS. Surfaceassociated Hsp60 chaperonin of Legionella pneumophila mediates invasion in a HeLa cell model. Infect Immunol 1998;66:4602-4610.

10. Huesca M, Borgia S, Hoffman PS, Lingwood CA. Acidic $\mathrm{pH}$ changes receptor binding specificity of Helicobacter pylori: a binary adhesion model in which surface heat shock (stress) proteins mediate sulfatide recognition in gastric colonization. Infect Immunol 1996;64:26432648.

11. Phadnis S, Parlow M, Levy M, et al. Surface localization of Helicobacter pylori urease and heat shock protein homolog requires bacterial autolysis. Infect Immunol 1996; 64:905-912.

12. Yamaguchi H, Osaki T, Kurihara N, et al. Heat-shock protein 60 homologue of Helicobacter pylori is associated with adhesion of $H$. pylori to human gastric epithelial cells. J Med Microbiol 1997;46:825-831.
13. Kamiya S, Yamaguchi H, Osaki T, Taguchi H. A virulence factor of Helicobacter pylori: role of heat shock protein in mucosal inflammation after $H$. pylori infection. J Clin Gastroenterol 1998;27(suppl 1):S35-39.

14. Segal G, Shuman HA. How is the intracellular fate of the Legionella pneumophila phagosome determined? Trends Microbiol 1998;6:253-255.

15. Andrews HL, Vogel JP, Isberg RR. Identification of linked Legionella pneumophila genes essential for intracellular growth and evasion of the endocytic pathway. Infect Immunol 1998;66:950-958.

16. Garduno RA, Quinn FD, Hoffman PS. HeLa cells as a model to study the invasiveness and biology of $L e$ gionella pneumophila. Can J Microbiol 1998;44:430-440.

17. Fernandez RC, Logan SM, Lee SHS, Hoffman PS. E1evated levels of Legionella pneumophila stress protein Hsp60 early in infection of human monocytes and L929 cells correlates with virulence. Infect Immunol 1996;64: 1968-1976.

18. Retzlaff C, Yamamoto Y, Okubo S, Hoffman PS, Friedman H, Klein TW. Legionella pneumophila-heat shock protein induced increase of interleukin-1b mRNA involves protein kinase $\mathrm{C}$ signaling in macrophages. Immunology 1996;89:281-288.

19. Skeen MJ, Miller MA, Shinnick TM, Ziegler HK. Regulation of murine macrophage IL-12 production. J Immunol 1996;56:1196-1206.

20. Huesca M, Goodwin A, Bhagwansingh A, Hoffman PS, Lingwood CA. Characterization of an acidic-pHinducible stress protein (hsp70), a putative sulfatide binding adhesin, from Helicobacter pylori. Infect Immunol 1998;66:4061-4067.

21. Benkirane R, Guinet R, Delaunay T. Purification and immunological studies of the cross-reaction between the 65-kilodalton gonococcal parietal protein and an antigen common to a wide range of bacteria. Infect Immunol 1992;60:3468-3471.

22. Frisk A, Ison CA, Lagergard T. GroEL heat shock protein of Haemophilus ducreyi: association with cell surface and capacity to bind to eukaryotic cells. Infect Immunol 1998;66:1252-1257.

23. Macellaro A, Tujulin E, Hjalmarsson $K$, Norlander L. Identification of a 71-kilodalton surface-associated Hsp70 homologue in Coxiella burnetii. Infect Immunol 1998;66:5882-5888. 


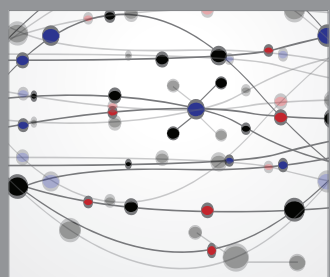

The Scientific World Journal
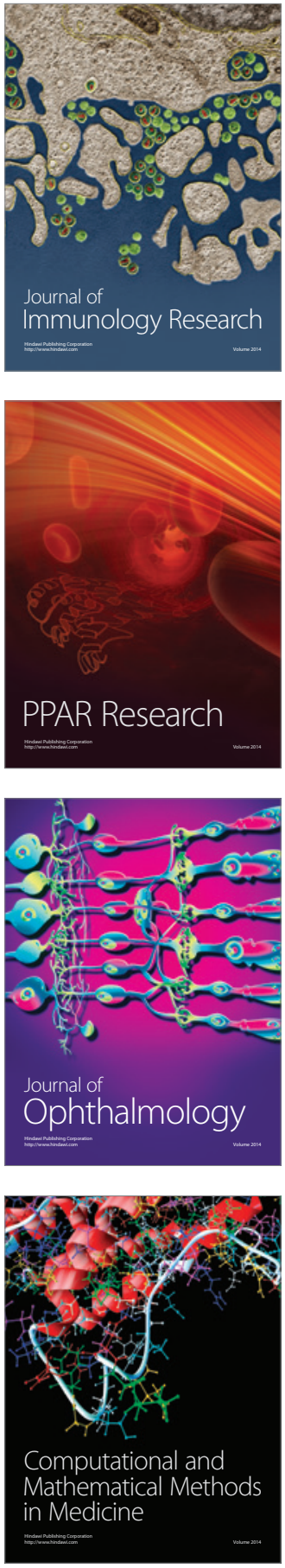

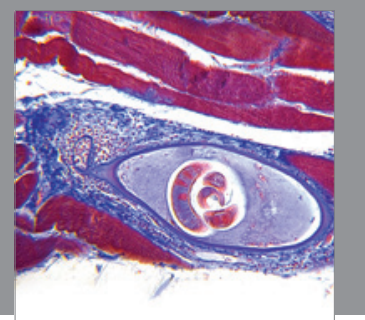

Gastroenterology

Research and Practice
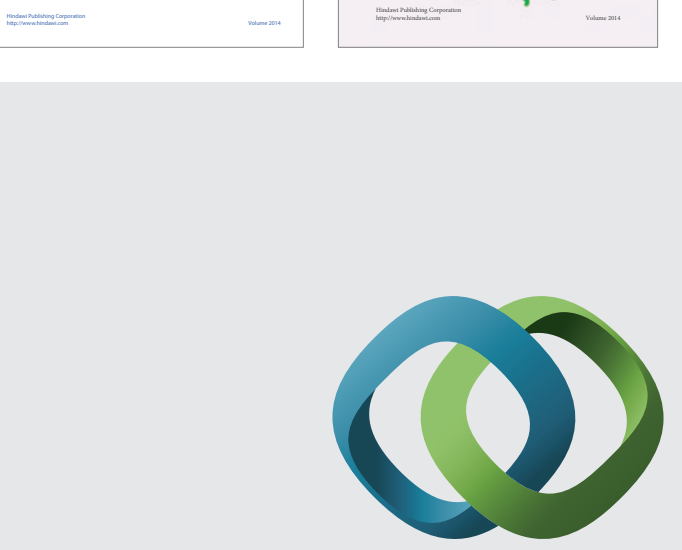

\section{Hindawi}

Submit your manuscripts at

http://www.hindawi.com
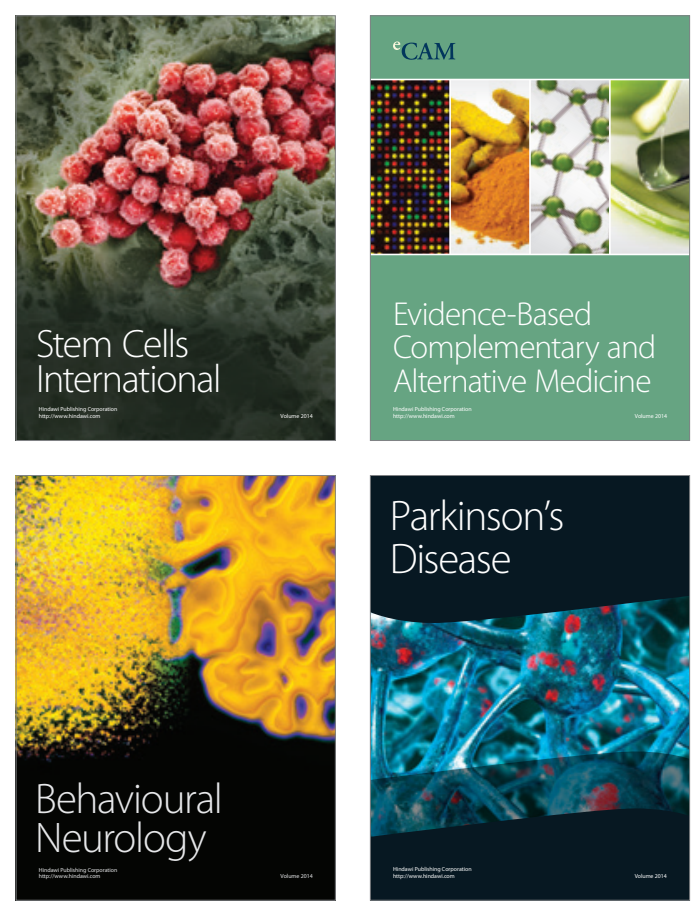

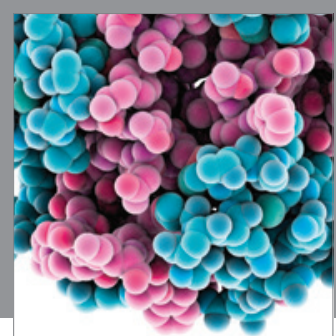

Journal of
Diabetes Research

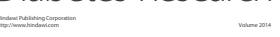

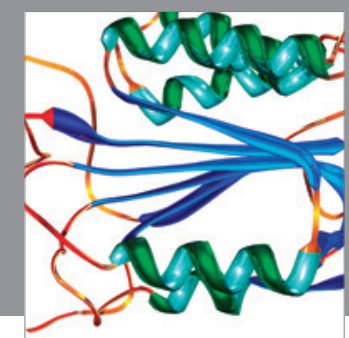

Disease Markers
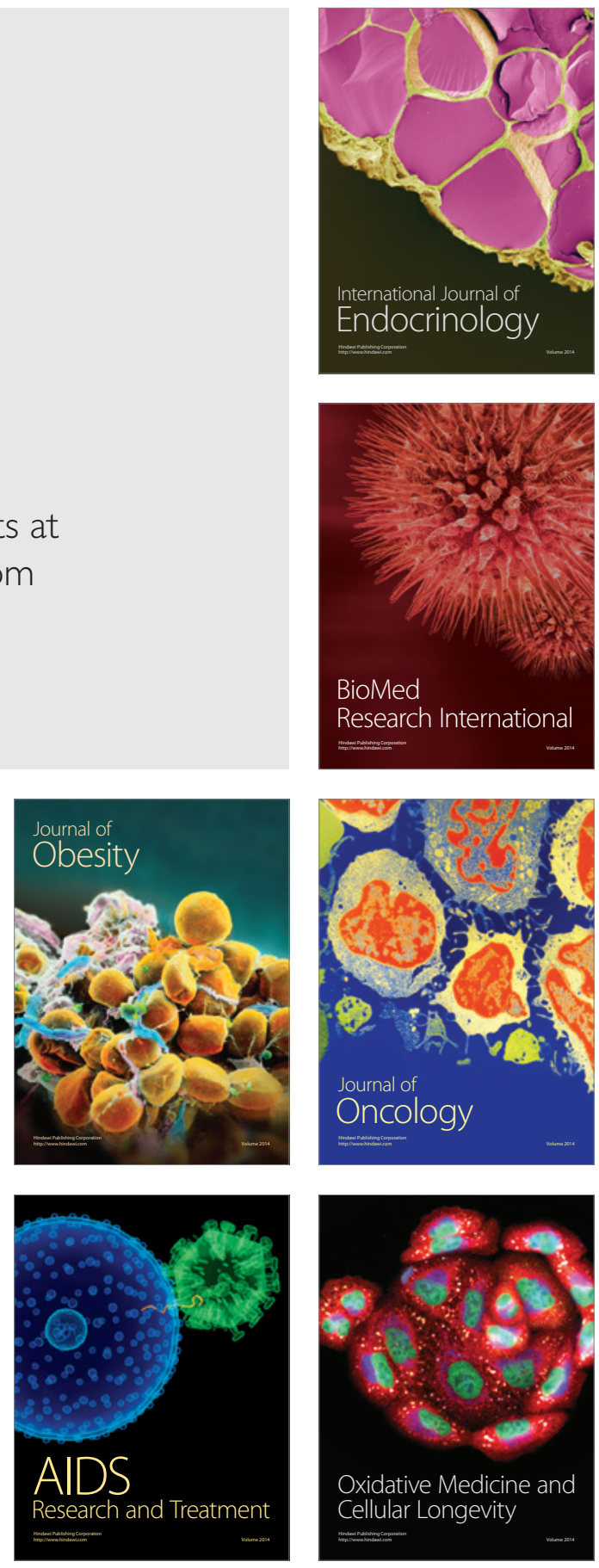